\title{
Numerical Study of the Eulerian Correlations and Lagrangian Velocity in Stably Stratified Homogeneous Turbulence
}

\author{
Abdallah Jaouabi and Taieb Lili \\ Laboratory of Fluid Mechanics, Department of Physics, \\ Faculty of Science of Tunis, University of Tunis El Manar, Tunisia
}

Received 2013-04-30, Revised 2013-05-23; Accepted 2013-05-28

\begin{abstract}
This study is based on a linear analysis in spectral space. To express the correlation double speed using Lagrangian Dual Eulerian correlations, we use the Corrsin assumption and then we compute the components of the tensor of the square displacements means. We begin by rewriting the basic linear equations governing the evolution of double correlations spectra. Subsequently, we analyze the numerical results.
\end{abstract}

Keywords: Turbulence Homogeneous Turbulent Diffusion, Stable Stratification, Corrsin Hypothesis

\section{INTRODUCTION}

In the context of a stably stratified homogeneous turbulence, (Kaneda and Ishida, 2000) presented explicit solutions of the linearized equations governing the evolution of such turbulence in spectral space. In the case of stable stratification and a Prandtl number $\operatorname{Pr}=1$, these solutions are in a form substantially easier. Such solutions allow us to express such double correlations speed and temperature at two points. It should be noted that the linear approximation is justified when the Froude number is small enough (or when the frequency of Brunt-Väisälä $\mathrm{N}$ is large enough), in these conditions, the nonlinear effects are small. The validity of these linear solutions was examined by (Kaneda and Ishida, 2000) by comparison to the results of DNS. It is important to remember that the covariance of displacement of a particle is expressed using Lagrangian velocity correlations. Intensive studies, both theoretical and experimental have been devoted to such correlations. (Kaneda and Ishida, 2000) then present an approximation model correlations Lagrangian speeds based on the approximation of Corrsin. In fact, in their numerical study on the removal of the vertical diffusion, these authors admit, for simplicity, the equivalence between the correlations speeds (two times) Lagrangian and Eulerian, this corresponds to a classical hypothesis called simplified Corrsin hypothesis. They then express the tensor component of displacement, the characteristic component of vertical diffusion, show that it has a constant term (independent of time) and a term representing a sum of damped distinct oscillations decrease over time because of phase mixture. The constant term, in the case of initial homogeneous isotropic turbulence energy potential zero, is $\frac{E_{\text {kin }}}{2 \mathrm{~N}^{2}}$ where $\mathrm{E}_{\text {kin }}$ is the initial kinetic energy. It should be noted that the explicit solutions of the linearized equations governing the evolution of the velocity spectra of scalar and presented by (Kaneda and Ishida, 2000) involving a coefficient $\alpha$, if the Prandtl number Pr is different from $1, \alpha^{2}$ can become negative, $\alpha$ becomes complex and a problem arises in the calculation of spectra double correlations speed two times. As part of the numerical study we present in this study, the approach is to calculate correlations cospectres double in two minutes with a direct numerical integration of the linear equations of evolution of these cospectres, the problem mentioned (Kaneda and Ishida, 2000) does not arise in the context of this study, which does not require a priori University of Tunis El Manar, Tunisia 
knowledge and the inclusion of explicit linear solutions. (Nicolleau and Vassilicos, 2000) studied the turbulent diffusion one and two particles in stably stratified homogeneous flow corresponding to high Reynolds numbers and low values of the Froude number. A Lagrangian model was developed using a Lagrangian Kinematic Simulation (KS Kinematic Simulation) solutions based on the Eulerian linear equations (assuming Boussinesq), valid for small values of the Froude number.

In this study, we propose, in the context of a stably stratified homogeneous turbulence using the Corrsin hypothesis to express the Lagrangian dual correlations speed using double speeds Eulerian correlations. After recalling the basic linear equations governing the evolution of velocity fluctuations and temperature in the spectral space, we write the evolution equations spectra correlations in two double points. The numerical solution of differential system thus obtained is used to determine, under the Corrsin assumption and an in iterative process, the components of the tensor of displacement means.

\subsection{Basic Linear Equations}

We consider a homogeneous turbulent flow, with stable stratification in the vertical direction (axis $x_{3}$ ). The linearized equations of evolution of velocity fluctuations $\mathrm{u}_{\mathrm{i}}(\overrightarrow{\mathrm{k}}, \mathrm{t})$ and temperature $\theta(\overrightarrow{\mathrm{k}}, \mathrm{t})$ occur in spectral space as follows (Anazaki and Hunt, 1996) Equation 1 and 2:

$$
\begin{aligned}
& \frac{\partial}{\partial t} u_{i}(\vec{k}, t)=-v k^{2} u_{i}(\vec{k}, t)-\left[\delta_{i 3}-\frac{k_{i} k_{3}}{k^{2}}\right] \alpha g \theta(\vec{k}, t) \\
& \frac{\partial}{\partial t} \theta(\vec{k}, t)=-\chi k^{2} \theta(\vec{k}, t)-\frac{N^{2}}{\alpha g} u_{3}(\vec{k}, t)
\end{aligned}
$$

$v$ and $\chi$ are respectively the kinematic viscosity and the thermal diffusivity. $g$ is the acceleration of gravity, $\alpha$ is the rate of volume expansion and $\mathrm{N}$ is the BruntVäisälä frequency: $\mathrm{N}^{2}=\alpha \mathrm{g} \frac{\partial \mathrm{T}}{\partial \mathrm{x}_{3}}$ where $\mathrm{T}$ is the average temperature. To achieve our goal, we made the choice to numerically solve the linearized equations of correlations at two instants $t$ and $t$ '(Jaouabi and Lili, 2011).

These equations involve the Prandtl number $\operatorname{Pr}=\frac{v}{\chi}$, Froude number $\operatorname{Fr}=\frac{\mathrm{u}_{0} \mathrm{k}_{\mathrm{p}}}{\mathrm{N}}$ and Reynolds number $\mathrm{Re}=\frac{\mathrm{u}_{0}}{\mathrm{vk}_{\mathrm{p}}}$.

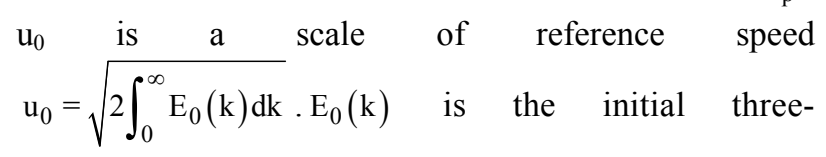

dimensional spectrum of kinetic energy. Is the wave number corresponding to the maximum of $\mathrm{E}_{0}(\mathrm{k})$ (Jaouabi and Lili, 2011)

\subsection{Presentation and Analysis of Results on the Evolution Correlations Eulerian and Lagrangian Velocity}

Kaneda and Ishida (2000) have determined the evolution of Eulerian and Lagrangian correlations velocities for 3 Values of $\operatorname{Fr}(0,19 ; 0,59 ; 1,87)$ corresponding respectively to values of $3 \mathrm{~N}^{2}(1000 ; 100$; 10), from explicit linear solutions (RDT) and Direct Numerical Simulations (DNS). It is in fact Eulerian and Lagrangian correlation standardized $\frac{\left\langle\mathrm{u}_{3}(\overrightarrow{\mathrm{x}}, \mathrm{t}) \mathrm{u}_{3}(\overrightarrow{\mathrm{x}}, 0)>\right.}{\mathrm{u}_{3}^{2}(\overrightarrow{\mathrm{x}}, 0)}$ and $\frac{\left\langle\mathrm{v}_{3}(\overrightarrow{\mathrm{x}}, \mathrm{t}) \mathrm{v}_{3}(\overrightarrow{\mathrm{x}}, 0)\right\rangle}{\mathrm{v}_{3}^{2}(\overrightarrow{\mathrm{x}}, 0)}$. The linear approach (RDT) that we have adopted in this study and the numerical code we developed to determine the correlation of Eulerian velocities, the latter, we obtain the Lagrangian correlations using the Corrsin hypothesis (the convergence of the iterative process introduced in the numerical code). In Fig. 1-3, we present the temporal evolution of these correlations to values equal to respectively 0,19, 0,59 and 1,87. We begin with Fig. 1 corresponding to $\mathrm{Fr}=0,19$ and $\mathrm{N}^{2}=1000$. Figure 1a shows the evolution of the normalized Eulerian correlation $\frac{<\mathrm{u}_{3}(\overrightarrow{\mathrm{x}}, \mathrm{t}) \mathrm{u}_{3}(\overrightarrow{\mathrm{x}}, 0)>}{\mathrm{u}_{3}^{2}(\overrightarrow{\mathrm{x}}, 0)}$. The solid curves from this study linear (RDT) RDT results of Kaneda and Ishida (2000) are practically merged, the agreement is likely to validate our part and RDT calculation code. The results of DNS (Kaneda and Ishida, 2000) (dashed line) have a gap that is growing over time with the RDT results.

This difference is obviously of the nonlinear effects. Figure 1b shows the evolution of the normalized Lagrangian correlation:

$$
\frac{<\mathrm{v}_{3}(\overrightarrow{\mathrm{x}}, \mathrm{t}) \mathrm{v}_{3}(\overrightarrow{\mathrm{x}}, 0)>}{\mathrm{v}_{3}^{2}(\overrightarrow{\mathrm{x}}, 0)}
$$

The dotted curve relates to the results of RDT (Kaneda and Ishida, 2000). The two curves practically combined in solid line represents the results of DNS (Kaneda and Ishida, 2000) and our results deduced from Eulerian correlations from a calculation RDT under Corrsin hypothesis. 
Abdallah Jaouabi and Taieb Lili / American Journal of Applied Sciences 10 (5): 460-465, 2013

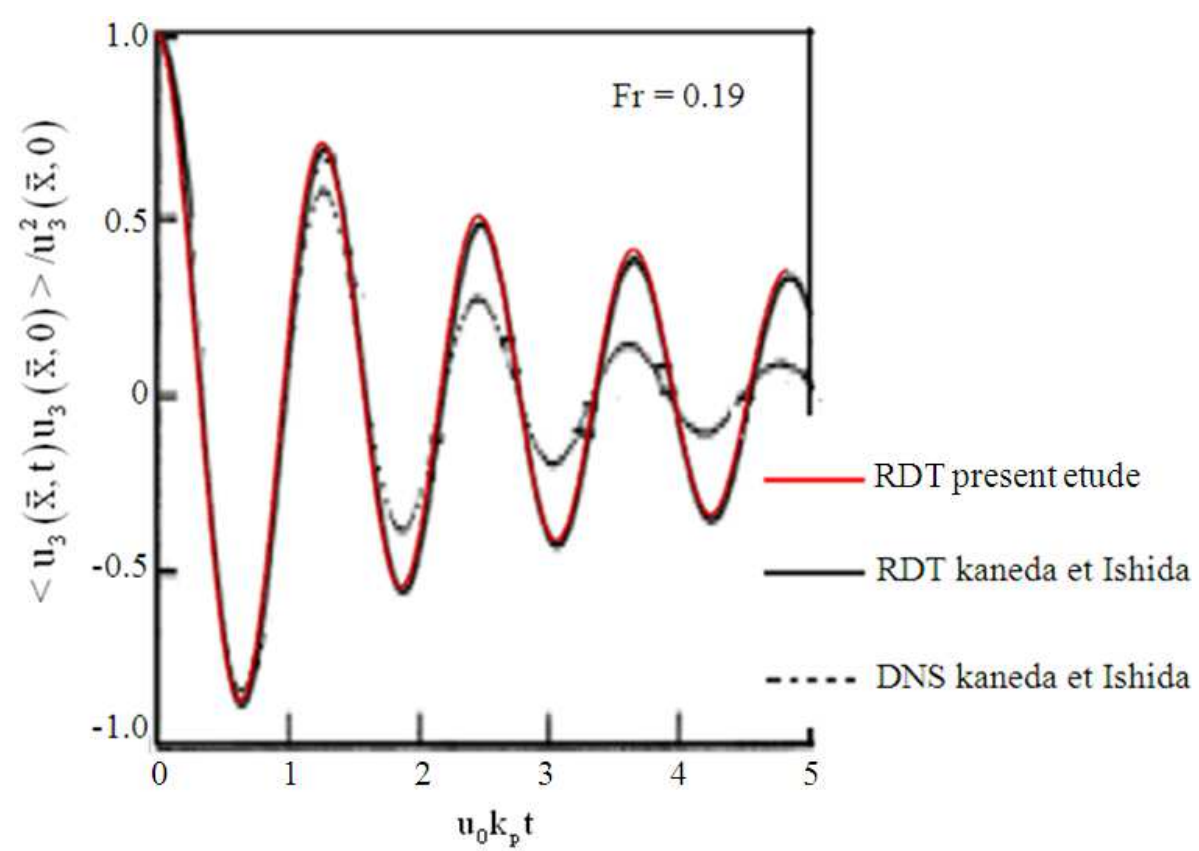

(a)

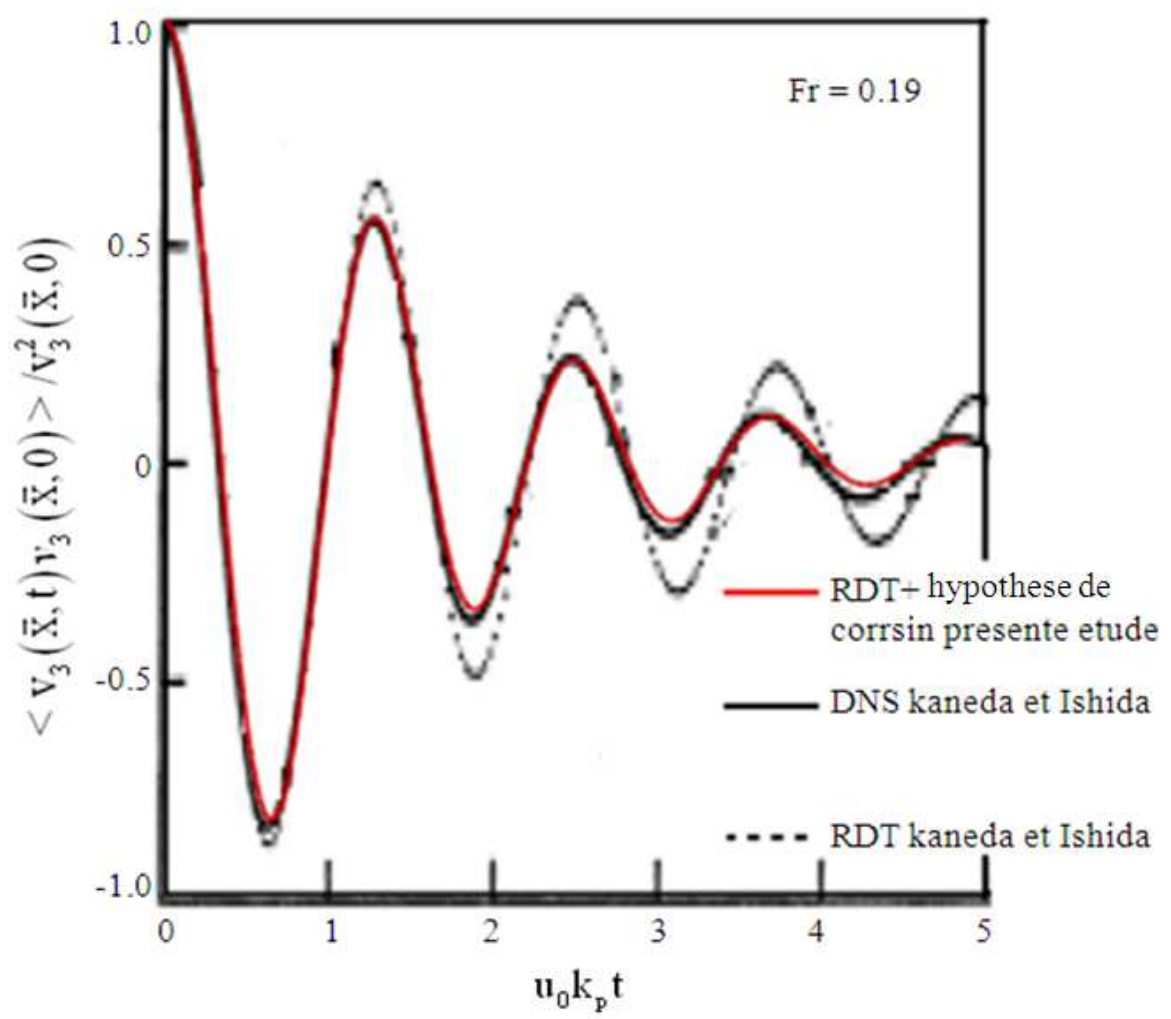

(b)

Fig. 1. Evolution of correlations eulerian and lagran-gian velocity normalized $\mathrm{Fr}=0,19\left(\mathrm{~N}^{2}=1000\right)$ 
Abdallah Jaouabi and Taieb Lili / American Journal of Applied Sciences 10 (5): 460-465, 2013

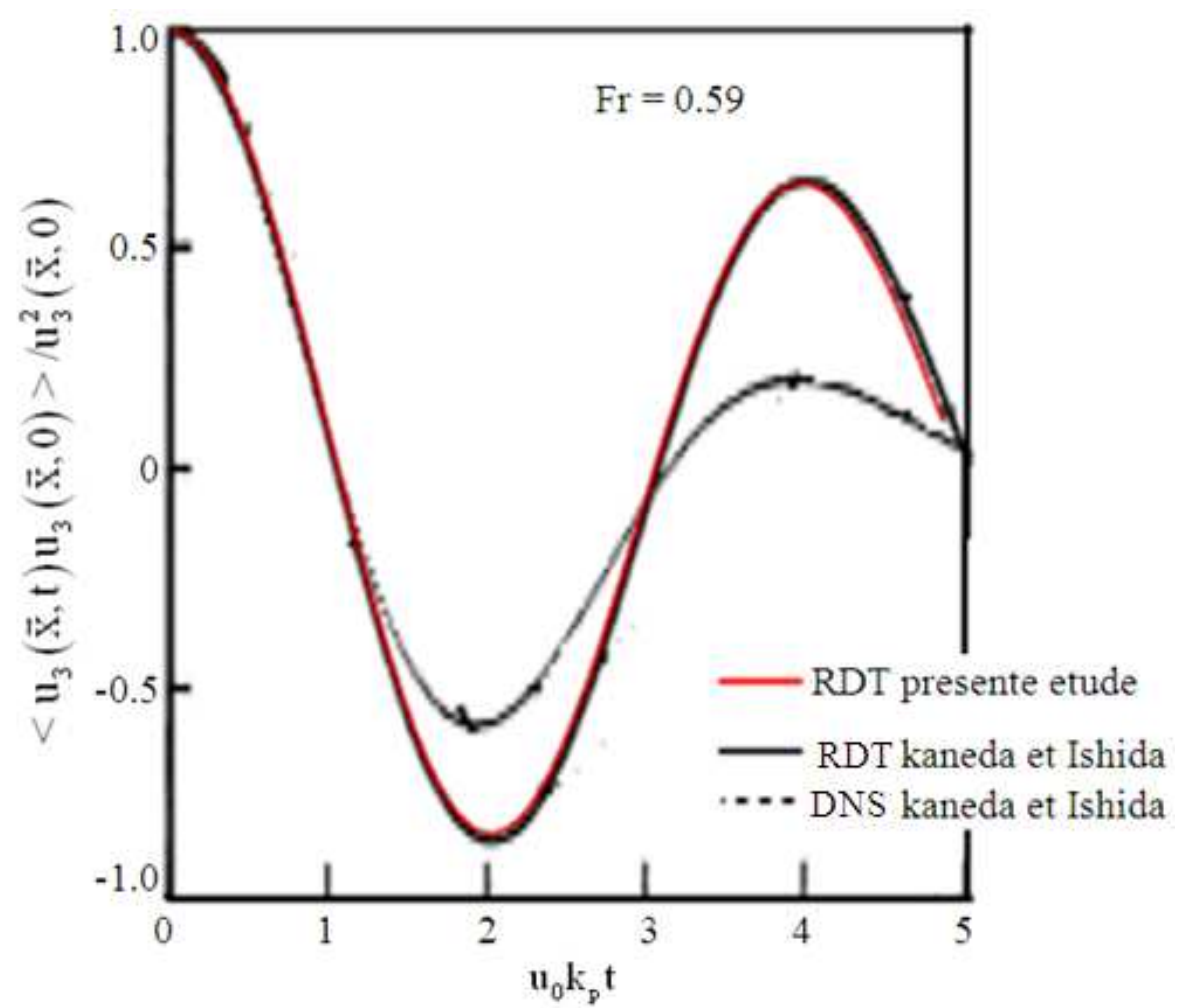

(a)

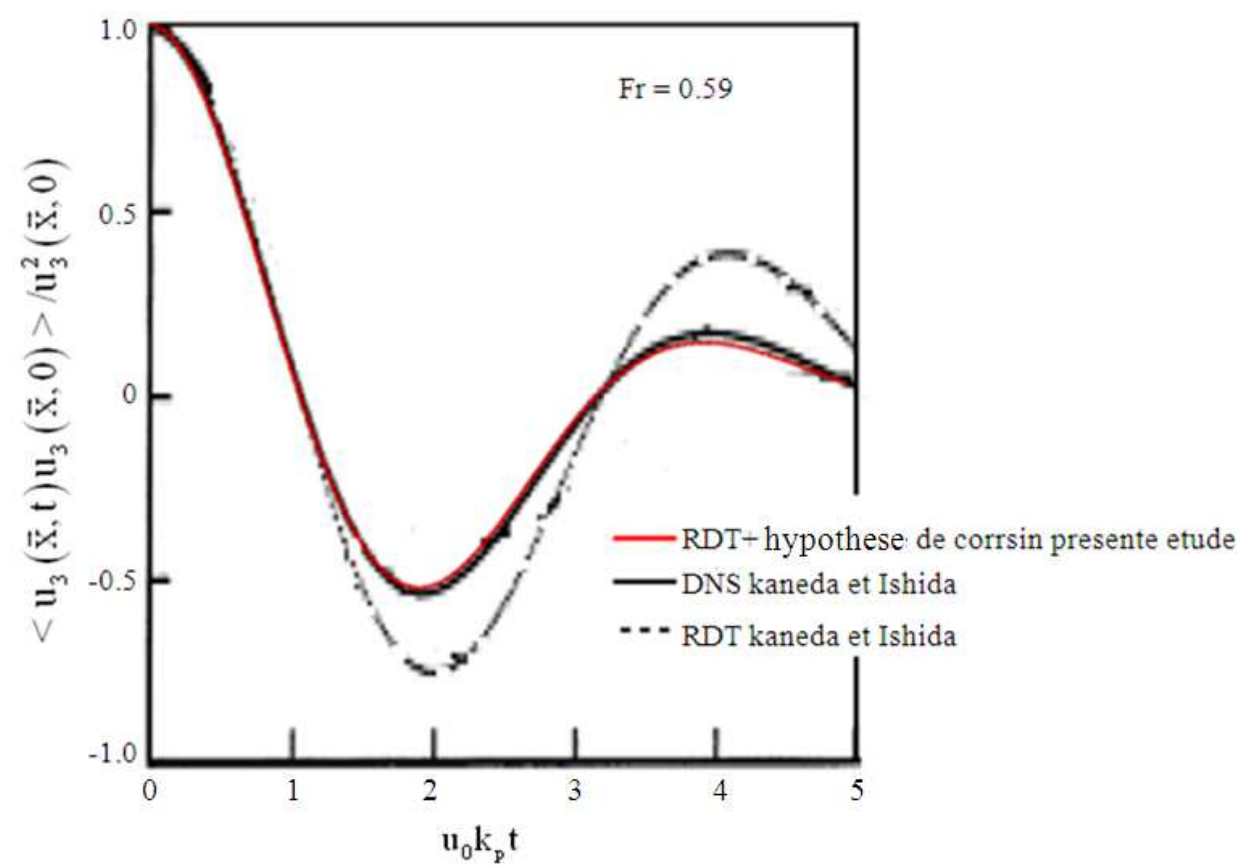

(b)

Fig. 2. Evolution of correlations eulerian and lagran-gian velocity normalized $\mathrm{Fr}=0,59\left(\mathrm{~N}^{2}=100\right)$ 


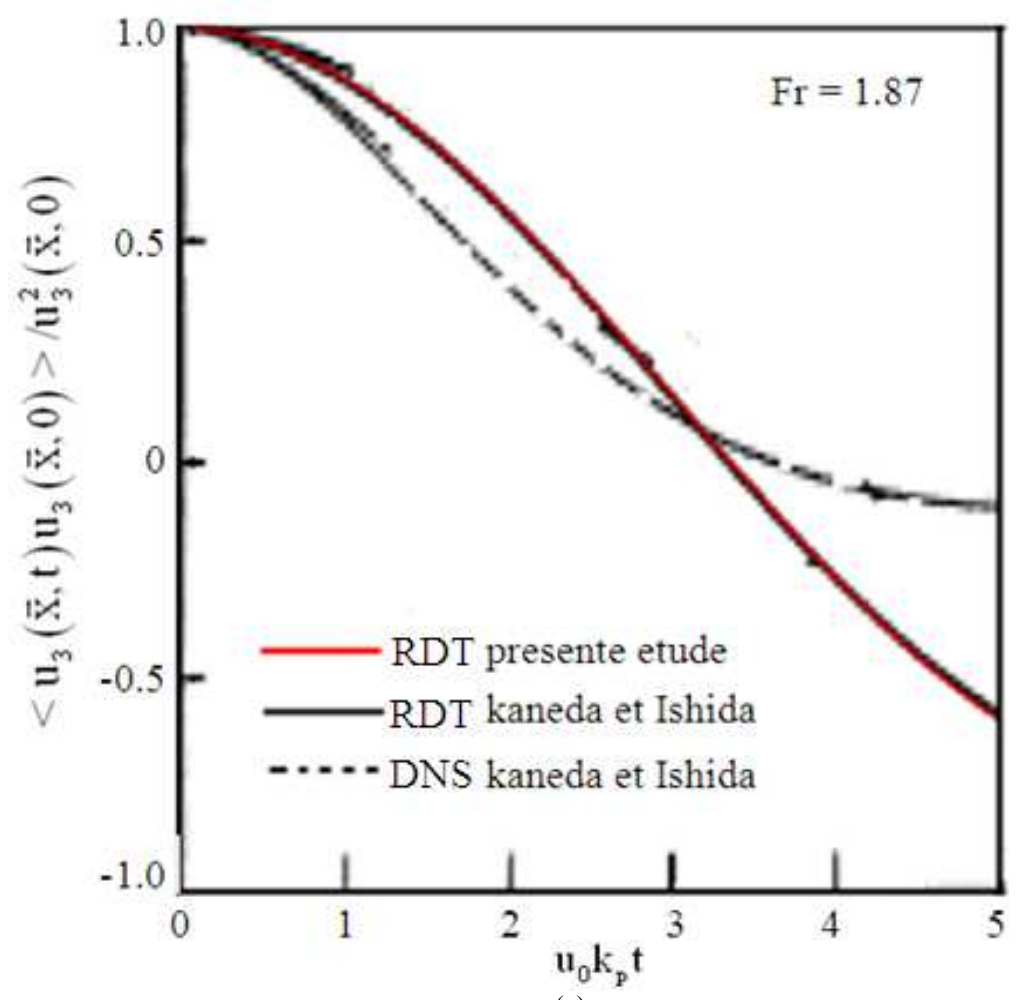

(a)

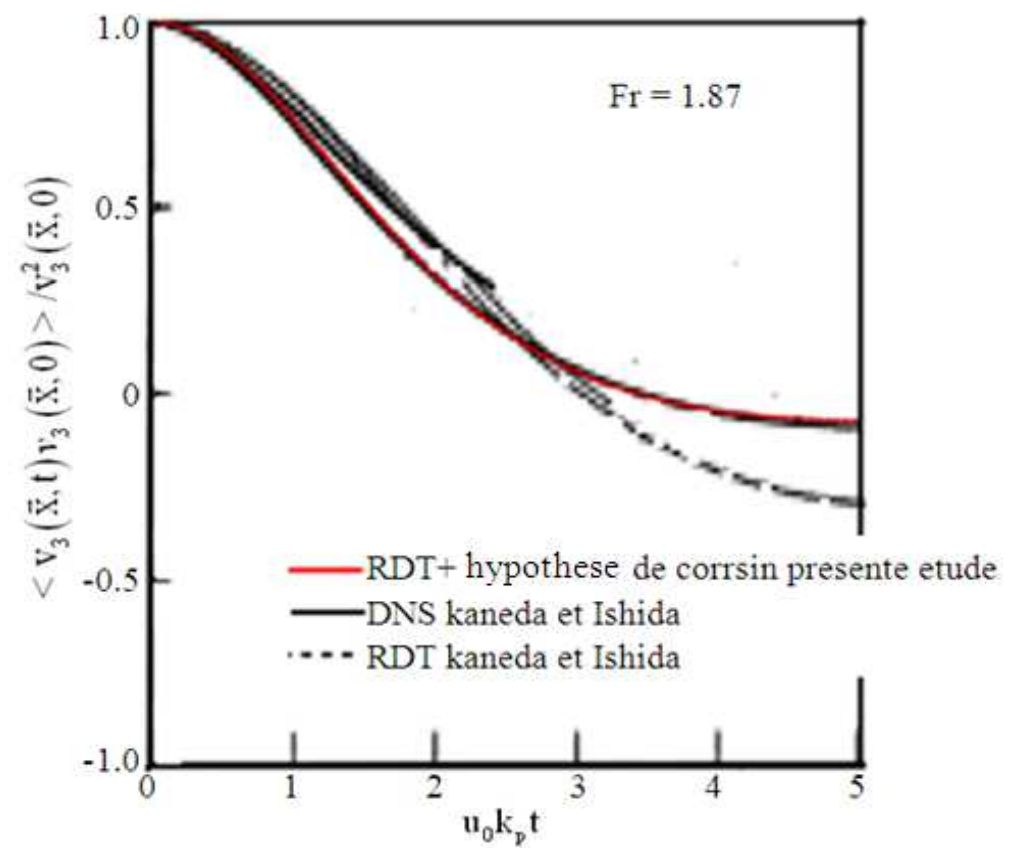

(b)

Fig. 3. Evolution of correlations eulerian and lagran-gian velocity normalized $\mathrm{Fr}=1,87\left(\mathrm{~N}^{2}=10\right)$ 
This important result suggests that nonlinear effects are not a significant contribution to the evolution of Lagrangian correlations when they are present in the evolution of Eulerian correlations. This important result also confirms the validity of the assumption of homogeneous turbulence Corrsin for a stably stratified.

The same comments to prevail $\mathrm{Fr}=0,59$ ( $2 \mathrm{a}$ and 2b) and $\mathrm{Fr}=1,87$ ( $3 \mathrm{a}$ and $3 \mathrm{~b}$ ). It is important to note that the stimulation time is too short to achieve a fully developed turbulence for $\mathrm{Fr}=1,87$ in particular. Noted finally that the nonlinear effects are much weaker than is Fr small $\left(\mathrm{N}^{2}\right.$ large $)$.

\section{CONCLUSION}

In this study, we numerically studied the case of a stably stratified homogeneous turbulence. This study is based on a linear analysis in spectral space. The essential option of this study is the use of the Corrsin hypothesis (associated with a normal distribution of Lagrangian velocity) to calculate dual Lagrangian velocity correlations from dual Eulerian velocities.

Finally, an agreement is observed between the results of the Lagrangian velocity correlations that we obtained by applying the Corrsin hypothesis and the results of DNS of (Kaneda and Ishida, 2000). This agreement reveals two important results: The validity of the Corrsin hypothesis for stably stratified homogeneous turbulence and insignificant contribution of non-linear effects in the evolution of Lagrangian velocity correlations.

\section{REFERENCES}

Anazaki, H.H. and J.C.R. Hunt, 1996. Linear processes in unsteady stably stratified turbulence. J. Fluid Mech., 318: 303-337. DOI: $10.1017 / \mathrm{S} 0022112096007136$

Jaouabi, A. and T. Lili, 2011. Numerical study of turbulent diffusion in homogeneous stably stratified turbulence using linear analysis. Comptes Rendus Mecanique, 339: 616-623.

Kaneda, Y. and T. Ishida, 2000. Suppression of vertical diffusion in strongly stratified turbulence. J. Fluid Mech., 402: 311-327. DOI: 10.1017/S0022112099007041

Nicolleau, F. and J.C. Vassilicos, 2000. Turbulent diffusion in stably stratified non-decaying turbulence. J. Fluid Mech., 410: 123-146.DOI: $10.1017 / \mathrm{S} 0022112099008113$ 